\title{
Measuring Resemblance of Complex Patterns
}

\author{
Michiel Hagedoorn ${ }^{\star}$ and Remco Veltkamp \\ Department of Computer Science, Utrecht University \\ Padualaan 14, $3584 \mathrm{CH}$ Utrecht, The Netherlands \\ mh@cs.uu.nl Remco.Veltkamp@cs.uu.nl
}

\begin{abstract}
On a collection of subsets of a space, fundamentally different metrics may be defined. In pattern matching, it is often required that a metric is invariant for a given transformation group. In addition, a pattern metric should be robust for defects in patterns caused by discretisation and unreliable feature detection. Furthermore, a pattern metric should have sufficient discriminative power. We formalise these properties by presenting five axioms. Finding invariant metrics without requiring such axioms is a trivial problem. Using our axioms, we analyse various pattern metrics, including the Hausdorff distance and the symmetric difference. Finally, we present the reflection metric. This metric is defined on finite unions of $n-1$-dimensional hyper-surfaces in $\mathbb{R}^{n}$. The reflection metric is affine invariant and satisfies our axioms.
\end{abstract}

\section{Introduction}

For any collection of patterns, subsets of a space, there exist many possible metrics. In pattern matching applications, it is often desired that such a metric is invariant for a given transformation group. The shape of a pattern can be defined as the pattern modulo the action of this invariance group. By minimisation of a pattern metric under the invariance group, one obtains a metric on shapes, see Rucklidge [13. If to be used in pattern matching, a pattern metric should be robust for the effects that occur with discretisation, see Fig. 1(a). In addition, a metric should have sufficient discriminative power.

Many pattern matching algorithms use a metric on simple closed curves. An important example is the Fréchet distance, see Alt and Godau [3]. Other pattern metrics for boundary curves are based on turning angle, see Cohen and Guibas [5], or normalised affine arc-length, see Huttenlocher and Kedem [10].

The Hausdorff metric is defined on the collection of all (non-empty) closed, bounded subsets of a metric space. Some algorithms are based on this metric, see [4], 11], 1]. However, the Hausdorff metric is not robust with respect to certain types of noise. For example, outliers, i.e. isolated points lying far away from the other points, can cause a dramatic increase in the Hausdorff distance. The Hausdorff metric is invariant for the group of isometries. The partial Hausdorff distance is a non-metric variant of the Hausdorff metric that is more robust

\footnotetext{
* supported by Philips Research
} 
for noise, see [12], 7]. The partial Hausdorff distance depends on a parameter estimating the amount of distortion.

For solid patterns, robust affine invariant metrics exist. Examples include the normalised volume of symmetric difference, and the difference of normalised indicators. The volume of symmetric difference was previously used by Alt et al. 2]. We introduced the difference of normalised indicators in [8].

Until now, little attention has been paid to affine invariant metrics on finite unions of curves in $\mathbb{R}^{2}$, or surfaces in $\mathbb{R}^{3}$. In this paper, we present the reflection metric. This metric is defined on finite unions of $(n-1)$-dimensional hypersurfaces in $\mathbb{R}^{n}$. The reflection metric is invariant under affine transformations. We show that the reflection metric is robust in many respects.

Section 2 presents axioms that express properties of pattern metrics, such as robustness. The general formulation allows the axioms to be verified for collections of patterns in any underlying topological space. Finding a metric satisfying a number of axioms becomes more difficult, or may even become impossible, as more general classes of patterns and larger invariance groups are desired.

Section 3 analyses various pattern metrics with respect to the domain of definition, the invariance group, and satisfaction of the axioms. We deal with the discrete metric, the Hausdorff metric, the (normalised) volume of symmetric difference, and the difference of normalised indicators. Lastly, we introduce the reflection metric.

\section{Axioms}

Definition 1. If $X$ is a topological space, $\mathcal{P}$ is a collection of subsets of $X$, and $d$ is a metric on $\mathcal{P}$, then we call the structure $(X, \mathcal{P}, d)$ a metric pattern space.

The elements of $\mathcal{P}$ will be called patterns. The value $d(A, B)$ is the distance between the patterns $A$ and $B$.

A collection of patterns $\mathcal{P}$ uniquely determines a maximal subgroup $T$ of the homeomorphisms $\operatorname{Hom}(X)$ under which $\mathcal{P}$ is closed. The subgroup $T$ consists of all $t \in \operatorname{Hom}(X)$ such that both the image $t(A)$ and the inverse image $t^{-1}(A)$ are members of $\mathcal{P}$ for all $A \in \mathcal{P}$.

The metric pattern space $(X, \mathcal{P}, d)$ is invariant for a transformation $g \in$ $T$ if $d(g(A), g(B))$ equals $d(A, B)$ for all $A, B \in \mathcal{P}$. The invariance group $G$ of a metric pattern space consists of all transformations in $T$ for which it is invariant. Figure $\mathbb{T}(\mathrm{b})$ shows patterns $A$ and $B$ in the Euclidean plane, and image patterns $g(A)$ and $g(B)$ under an affine transformation $g$. Invariance for affine transformations makes the distance between two patterns independent of the choice of coordinate system.

Below, we present axioms for metric pattern spaces. The first four axioms express various types of robustness. These robustness axioms are preserved if the collection of patterns is restricted, or the patterns are replaced with their complements. The fifth and last axiom is a property that can be verified for metric spaces in general, enforcing a mild form of discrimination. 


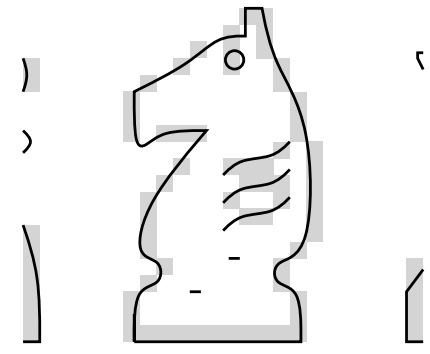

(a)

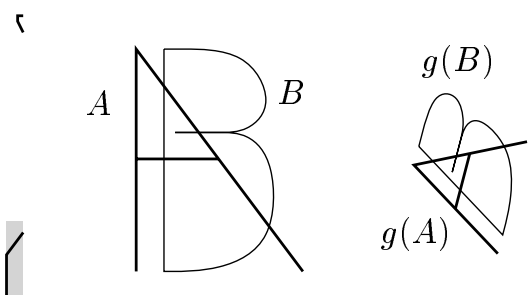

(b)

Fig. 1. (a) Discretisation effects (b) Invariance.

First, we discuss an axiom which states that transformations $t$ "close" to the identity, result in a "small" distance $d(A, t(A))$. In a topological space, this kind of closeness can be described by considering small open neighbourhoods of a point. For this purpose, we give the group of transformations $T$ the compactopen topology, which is defined in terms of the topology on $X$. This way, $T$ can be considered a topological space in which transformations are points, and each transformation has open neighbourhoods that are sets of transformations.

The compact-open topology is characterised by its collection of open sets. A collection of subsets $\mathcal{S}$ of a space $S$ whose union equals $S$, is called a subbasis for a topology on a space $S$. The topology generated by the subbasis $\mathcal{S}$ consists of all unions of finite intersections of elements of $\mathcal{S}$. The (relative) compact-open topology on the set of transformations $T$ is generated by the subbasis consisting of all sets of the form

$$
S(K, U)=\{t \in T \mid t(K) \subseteq U\}
$$

where $K \subseteq X$ is compact, and $U \subseteq X$ is open.

The metric pattern space $(X, \mathcal{P}, d)$ is called deformation robust if it satisfies the following axiom:

Axiom 1. For each $A \in \mathcal{P}$ and $\epsilon>0$, an open neighbourhood $I \subseteq T$ of the identity transformation exists such that $d(A, t(A))<\epsilon$ for all $t \in I$.

Deformation robustness is equivalent to saying that the map $t \mapsto t(A)$ with domain $T$ and range $\mathcal{P}$ is continuous, for each pattern $A \in \mathcal{P}$. Figure 2] a) shows the image of $A$ under a transformation $t$ contained in an open neighbourhood of the identity. This neighbourhood is a finite intersection of subbasis elements $S\left(K_{i}, U_{i}\right)$ generated by compact segments $K_{i}$ of $A$ and open balls $U_{i}$ containing $A_{i}$.

Define the boundary of a subset $A$ of $X$ by $\operatorname{Bd}(A)=\mathrm{Cl}(A)-\operatorname{Int}(A)$, where $\mathrm{Cl}(A)$ and $\operatorname{Int}(A)$ denote the closure and the interior of $A$ in $X$, respectively. We call a metric pattern space blur robust if the following axiom holds: 
Axiom 2. For each $A \in \mathcal{P}$ and $\epsilon>0$, an open neighbourhood $U$ of $\operatorname{Bd}(A)$ exists, such that $d(A, B)<\epsilon$ for all $B \in \mathcal{P}$ satisfying $B-U=A-U$ and $\operatorname{Bd}(A) \subseteq \operatorname{Bd}(B)$.

The axiom says that changes "close" to the boundary of $A$ do not cause discontinuities. This way, the axiom ensures that "shadow" or "double" edges do not significantly affect the distance between patterns. Figure 2(b) shows a neighbourhood $U$ of $\operatorname{Bd}(A)$ in which parts of $\operatorname{Bd}(B)$ occur that are not in $\operatorname{Bd}(A)$.

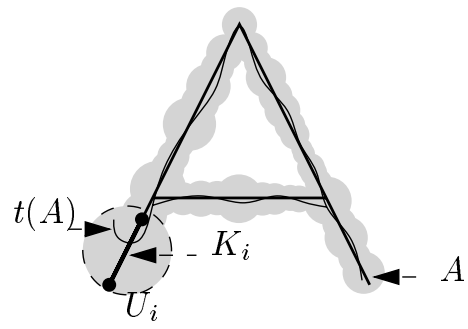

(a)

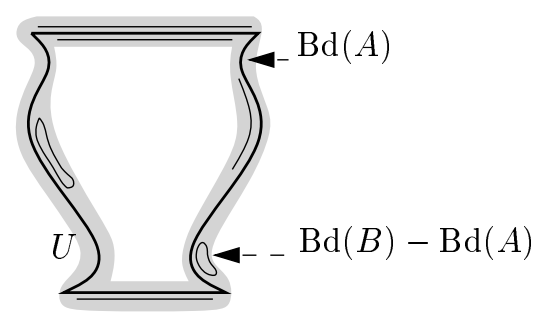

(b)

Fig. 2. (a) Deformation robustness (b) Blur robustness.

A crack of $A$ is a closed subset $R$ of $X$ consisting entirely of limit points of $\operatorname{Bd}(A)-R$. This means that all open neighbourhoods of a point $x \in R$ intersect $\operatorname{Bd}(A)-R$. Cracks are parts of the boundary, that can be "restored" after having been removed from the boundary, by taking the closure of the remaining boundary. Changing a pattern in neighbourhoods of a crack may cause the pattern (or its complement) to become separated or connected. We say $(X, \mathcal{P}, d)$ is crack robust if the next axiom holds:

Axiom 3. For each $A \in \mathcal{P}$, each crack $R$ of $A$, and $\epsilon>0$, an open neighbourhood $U$ of $R$ exists such that $A-U=B-U$ implies $d(A, B)<\epsilon$ for all $B \in \mathcal{P}$.

This axiom says that applying changes to $A$ within a small enough neighbourhood of a crack of $A$ results in a pattern $B$ close to $A$ in pattern space. Whether the connectedness is preserved does not matter.

Figure 3(a) shows a pretzel, consisting of two topological 1-spheres glued together at a point $x$. The set $R=\{x\}$ is a crack of $A$. This situation is similar to a saddle-point event encountered while varying the threshold during image segmentation.

If the following axiom is satisfied, we call a metric pattern space noise robust:

Axiom 4. For each $A \in \mathcal{P}, x \in X$, and $\epsilon>0$, an open neighbourhood $U$ of $x$ exists such that $B-U=A-U$ implies $d(A, B)<\epsilon$ for all $B \in \mathcal{P}$. 
This axiom says that changes do not cause discontinuities provided the changes happen within small regions. ¿From the triangle inequality it follows that an equivalent axiom is obtained when neighbourhoods of finite point sets instead of singletons are considered. In a practical sense, satisfaction of the axiom means that the metric is well-behaved with respect to "outliers" and "occlusion". Another interpretation is that the metric is robust for "emerging" or "disappearing" regions.

Figure 3(b) shows a pawn-like pattern $A$ and a point $x$. Addition of noise $B-A$ within a neighbourhood $U$ of $x$ results in a new pattern $B$. Axiom 4 says that we can make the distance between $A$ and $B$ smaller by making $U$ smaller.

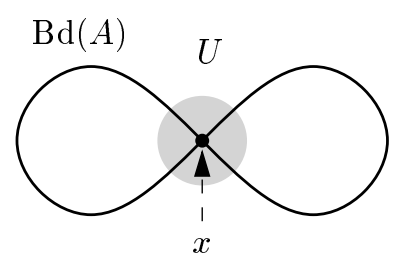

(a)

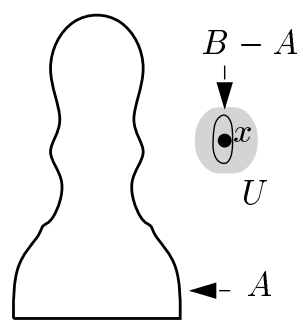

(b)

Fig. 3. (a) Crack robustness (b) Noise robustness.

Each of the previous axioms would still hold if we replaced the metric $d$ with a bounded metric $\rho(A, B)=\min (c, d(A, B))$ for any fixed $c>0$. The following axiom prohibits "cut-off" bounding of metrics in this manner. We say $(X, \mathcal{P}, d)$ is strictly increases if the following is true:

Axiom 5. For any $A, B \in \mathcal{P}, a C \in \mathcal{P}$ exists such that $d(A, C)>d(A, B)$.

This axiom expresses a form of discrimination: Given two patterns, there should always be a third pattern such that the distance between the first and the third pattern is larger than the distance between the first and the second pattern.

\section{Examples}

In this section, we analyse examples of metric pattern spaces, using the properties given in Section 2. In each example, we fix the space $X$ and the collection of patterns $\mathcal{P}$. Therefore, we will say that an axiom holds for a metric on $d$, when we actually mean that the axiom holds for the metric pattern space $(X, \mathcal{P}, d)$. In the following, we will discuss the discrete metric, the Hausdorff metric, the volume of symmetric difference, the normalised volume of symmetric difference, the difference of normalised indicators. After that, we present the reflection metric. 


\subsection{Discrete Metric}

The following example, the discrete metric, illustrates that it is no problem finding a metric defined on any collection of patterns invariant under any group of homeomorphisms in a topological space $X$. Only demanding the satisfaction of axioms, makes the problem of finding "invariant" metrics non-trivial.

Let the collection of patterns be $\wp\left(\mathbb{R}^{n}\right)$, the power set of $\mathbb{R}^{n}$.

Definition 2. The discrete metric on $\wp\left(\mathbb{R}^{n}\right)$ is given by

$$
d_{\mathrm{D}}(A, B)=\left\{\begin{array}{ll}
0 & \text { if } A=B \\
1 & \text { otherwise }
\end{array} .\right.
$$

The discrete metric is invariant for all homeomorphisms. However, this metric satisfies none of our axioms. Below, we provide simple examples contradicting each of the axioms.

Deformation robustness does not hold because non-empty open subsets of $\mathbb{R}^{n}$ always contain more than one point. Choose $A=\{x\}$, where $x$ is an arbitrary point in $\mathbb{R}^{n}$, and choose $\epsilon=1$. Then, $d(A, t(A))<\epsilon$ if and only if $t(x)=x$. It can be shown that any open $I$ containing the identity, contains a transformation $t$ for which $t(x) \neq x$, implying $d(A, t(A))=1$.

The following example contradicts blur robustness. Choose $A=\{x\}$ and $\epsilon=1$. For any neighbourhood $U$ of $x$, choose $B=\{y, x\}$, where $y \neq x$ is in $U$. Then $A \neq B$, so $d_{\mathrm{D}}(A, B)=1$.

Using the unit sphere $S^{1}$ in $\mathbb{R}^{2}$, a counterexample for crack robustness can be constructed. Choose $A=S^{1}$, and let $R$ consist of a single point on $S^{1}$. Clearly $R \subseteq \mathrm{Cl}(\operatorname{Bd}(A)-R)$. Now let $\epsilon=1$. For any open neighbourhood $U$ of $R$, choose $B=A-R$, giving $d_{\mathrm{D}}(A, B)=1$.

A counterexample for noise robustness is obtained by choosing $A=\{x\}$ and $\epsilon=1$. For any neighbourhood of $U$, we can always choose $B=\varnothing$ giving $d_{\mathrm{D}}(A, B)=1$.

Agreeing with intuition, the discrete metric is not strictly increasing. The distance between any two distinct patterns always equals one.

\subsection{Hausdorff Metric}

The Hausdorff metric is defined on the collection of (non-empty) bounded, closed subsets of any given metric space $X$. Here, we consider the Hausdorff distance for Euclidean space $X=\mathbb{R}^{n}$. The collection of patterns $\mathcal{K}^{n}$ consists of all non-empty compact subsets of $\mathbb{R}^{n}$.

Let $B(x, \epsilon)$ denote the open Euclidean ball in $\mathbb{R}^{n}$ centred at $x \in \mathbb{R}^{n}$ with radius $\epsilon>0$. For any subset $S$ of $\mathbb{R}^{n}$, define the $\epsilon$-neighbourhood of $S$ in $\mathbb{R}^{n}$ as

$$
S^{\epsilon}=\bigcup_{x \in S} B(x, \epsilon)
$$

The following definition uses these $\epsilon$-neighbourhoods. 
Definition 3. The Hausdorff metric on $\mathcal{K}^{n}$ is given by

$$
d_{\mathrm{H}}(A, B)=\inf \left\{\epsilon>0 \mid A \subseteq B^{\epsilon} \text { and } B \subseteq A^{\epsilon}\right\} .
$$

It is not difficult to show that the invariance group for the Hausdorff metric equals Iso $\left(\mathbb{R}^{n}\right)$, the group of isometries in $\mathbb{R}^{n}$.

The Hausdorff metric is deformation robust. Let $A \in \mathcal{K}^{n}$ and $\epsilon>0$ be given. Cover $A$ with a finite number of balls $\left\{B\left(c_{1}, \delta\right), \ldots, b\left(c_{k}, \delta\right)\right\}$ each having radius $\delta=\frac{1}{4} \epsilon$. Define an open set of transformations as a finite intersection of subbasis elements:

$$
I=\bigcap_{i=1}^{k}\left\{t \in \operatorname{Hom}\left(\mathbb{R}^{n}\right) \mid t\left(\operatorname{Cl}\left(B\left(c_{i}, \delta\right)\right)\right) \subseteq B\left(c_{i}, 2 \delta\right)\right\} .
$$

Clearly, $d_{\mathrm{H}}(A, t(A)) \leq 2 \delta<\epsilon$ for each $t \in I$.

We prove blur-robustness for the Hausdorff metric. Let $A \in \mathcal{K}^{n}$ and $\epsilon>0$. Choose $\delta=\frac{\epsilon}{2}$ and $U=\operatorname{Bd}(A)^{\delta}$. Suppose $B \in \mathcal{P}$ satisfies $B-U=A-U$ and $\operatorname{Bd}(A) \subseteq \operatorname{Bd}(B)$. Since $A \subseteq B^{\delta}$ and $B \subseteq A^{\delta}$, we have $d_{\mathrm{H}}(A, B) \leq \delta<\epsilon$.

The Hausdorff metric is crack robust. Let $A \subseteq \mathbb{R}^{n}$ be non-empty compact and $R$ be a crack of $A$. Set $\delta=\frac{1}{4} \epsilon$. Cover $R$ with a finite collection of balls centred at points of $R$, and having radius $\delta:\left\{B_{1}, \ldots, B_{k}\right\}$. For each ball $B_{i}$ choose a point $x_{i}$ in the intersection with $\operatorname{Bd}(A)-R$. Construct an open set containing $R$ by setting $U=\bigcup_{i=1}^{k} B_{i}-\left\{x_{1}, \ldots, x_{k}\right\}$. Then $d_{\mathrm{H}}(A, B) \leq 2 \delta<\epsilon$ for any $B \in \mathcal{S}^{n}$ satisfying $B-U=A-U$.

A counterexample for noise robustness can be constructed in the real line. Choose $A=\{0\}, x=1$, and $\epsilon=1$. For any neighbourhood $U$ of 1 , choosing $B=\{0,1\}$ gives $A-U=B-U$ and $d_{\mathrm{H}}(A, B)=\epsilon$.

The Hausdorff metric is strictly increasing. Let $A, B \in \mathcal{K}^{n}$. Let $\epsilon=d_{\mathrm{H}}(A, B)$. Choose a point $c \in \mathbb{R}^{n}$ such that $\inf _{x \in A}\|x-c\|>\epsilon$. Clearly $d_{\mathrm{H}}(A, B \cup\{c\})>\epsilon$.

\subsection{Volume of Symmetric Difference}

Let $V=\left\{v_{0}, \ldots, v_{k}\right\}$ be a pointwise independent subset of $\mathbb{R}^{n}$. The closed $k$-simplex determined by $V$ is the set of points in the $k$-dimensional hyper-plane containing $V$ having nonnegative barycentric coordinates with respect to $V$. Two simplices are properly joined if their intersection is a subsimplex of both simplexes. Define $\mathcal{S}^{n}$ as the collection of non-empty subsets of $\mathbb{R}^{n}$ that are $C^{1}$ diffeomorphic with a finite union of properly joined $n$-simplices.

For a compact set $A \subseteq \mathbb{R}^{n}$, let $\operatorname{vol}(A)$ denote the volume of $A$ which equals the Lebesgue-integral of the indicator $1_{A}$.

Definition 4. The volume of symmetric difference on $\mathcal{S}^{n}$ is given by

$$
d_{\mathrm{S}}(A, B)=\operatorname{vol}((A-B) \cup(B-A)) .
$$

The function $d_{\mathrm{S}}$ is obviously a semimetric. The fact that $d_{\mathrm{S}}$ is a metric on $\mathcal{S}^{n}$ follows from the fact that $\operatorname{Int}(A)-B$ is non-empty and open if $A$ is not a subset of $B$, and $A, B \in \mathcal{S}^{n}$. 
The collection of patterns $\mathcal{S}^{n}$ is closed under $\mathrm{C}^{1}\left(\mathbb{R}^{n}\right)$, the group of diffeomorphisms on $\mathbb{R}^{n}$. It can be shown that $d_{\mathrm{S}}$ has invariance group $\mathrm{UJ}\left(\mathbb{R}^{n}\right)$, the class of diffeomorphisms with unit magnitude Jacobi-determinant everywhere. These transformations preserve the volume of each set. The special affine transformations form a proper subgroup of $\mathrm{UJ}\left(\mathbb{R}^{n}\right)$

The collection of patterns $\mathcal{S}^{n}$ is defined such that for each $A \in \mathcal{S}^{n}$, an arbitrary small neighbourhood of the boundary of $A$ can be chosen: For each $\epsilon>0$, an open neighbourhood $U$ of $\operatorname{Bd}(A)$ exists with $\operatorname{vol}(U)<\epsilon$. This property is essential in proving the deformation, blur and crack robustness axioms.

The volume of symmetric difference is deformation robust. Let $A \in \mathcal{S}^{n}$ and $\epsilon>0$. Choose a neighbourhood $U$ of $\operatorname{Bd}(A)$ with volume strictly smaller than $\epsilon$. An open neighbourhood of the identity transformation is given by

$$
I=\left\{t \in \mathrm{C}^{1}\left(\mathbb{R}^{n}\right) \mid t(\operatorname{Bd}(A)) \subseteq U\right\} .
$$

Suppose $t \in I$. Since both $A-t(A)$ and $t(A)-A$ are contained in $U$, it follows that $d_{\mathrm{S}}(A, t(A)) \leq \operatorname{vol}(U)<\epsilon$.

The symmetric difference is blur robust. For given $A \in \mathcal{S}^{n}$ and $\epsilon>0$, choose some open neighbourhood $U$ of $\operatorname{Bd}(A)$ with volume smaller than $\epsilon$. Clearly, $d_{\mathrm{S}}(A, B)<\epsilon$ for any $B \in \mathcal{S}^{n}$ satisfying $B-U=A-U$.

We can also prove crack robustness by choosing neighbourhoods with sufficiently small volumes. For $A \in \mathcal{S}^{n}$, crack $R$ of $A$, and $\epsilon>0$, choose an open neighbourhood $U$ of $R$ with volume less than $\epsilon$. Any $B \in \mathcal{S}^{n}$ satisfying $B-U=A-U$ gives $d_{\mathrm{S}}(A, B)<\epsilon$.

Proving noise robustness is almost the same. Let $A \in \mathcal{S}^{n}, x \in \mathbb{R}^{n}$, and $\epsilon>0$. Choose a neighbourhood $U$ of $x$ with volume smaller than $\epsilon$, then $A-U=B-U$ implies $d_{\mathbf{S}}(A, B)<\epsilon$ for all $B \in \mathcal{S}^{n}$.

We show that the volume of symmetric difference is strictly increasing. Let $A, B \in \mathcal{S}^{n}$. Let $S$ be a closed $n$-simplex, disjoint with $A \cup B$. Then, $d_{\mathrm{S}}(A, B \cup S)>$ $d_{\mathrm{S}}(A, B)$.

\subsection{Normalised Volume of Symmetric Difference}

Definition 5. The normalised volume of symmetric difference on $\mathcal{S}^{n}$ is given by

$$
d_{\mathrm{NS}}(A, B)=\frac{\operatorname{vol}((A-B) \cup(B-A))}{\operatorname{vol}(A \cup B)}
$$

The function $d_{\mathrm{NS}}$ is a metric with invariance group $\mathrm{CJ}\left(\mathbb{R}^{n}\right)$, the class of diffeomorphisms with constant magnitude Jacobi-determinant everywhere. These transformations preserve the ratio of volume for any two sets. The affine transformations $\operatorname{Af}\left(\mathbb{R}^{n}\right)$ form a proper subgroup of $\operatorname{CJ}\left(\mathbb{R}^{n}\right)$.

For proving deformation, blur, crack and noise robustness, it suffices to show that the topology induced by the metric $d_{\mathrm{S}}$ is finer than that induced by that of $d_{\mathrm{NS}}$. This is done in the following simple lemma. 
Lemma 1. For each $A \in \mathcal{S}^{n}$ and each $\epsilon>0$, there exists a $\delta>0$ such that $d_{\mathrm{S}}(A, B)<\delta$ implies $d_{\mathrm{NS}}(A, B)<\epsilon$ for all $B \in \mathcal{S}^{n}$.

We are left with showing that $d_{\mathrm{NS}}$ is strictly increasing. For this purpose, the construction given for the volume of symmetric difference suffices.

\subsection{Difference of Normalised Indicators}

For $A \in \mathcal{S}^{n}$, define a function $\nu_{A}: \mathbb{R}^{n} \rightarrow \mathbb{R}$ by $\nu_{A}(x)=\operatorname{vol}(A)^{-1} 1_{A}(x)$.

Definition 6. The difference of normalised indicators $d_{\mathrm{NI}}$ for $\mathcal{S}^{n}$ is given by

$$
d_{\mathrm{NI}}(A, B)=\int_{\mathbb{R}^{n}}\left|\nu_{A}(x)-\nu_{B}(x)\right| d x .
$$

The invariance group of $d_{\mathrm{NI}}$ is $\mathrm{CJ}\left(\mathbb{R}^{n}\right)$.

The following lemma is the analogue of Lemma 1 for the difference of normalised indicators. It shows that the topology $d_{\mathrm{S}}$ induces on $\mathcal{S}^{n}$ is finer than that induced by $d_{\mathrm{NI}}$. This immediately gives deformation, blur, crack, and noise robustness for $d_{\mathrm{NI}}$.

Lemma 2. For each $A \in \mathcal{S}^{n}$ and each $\epsilon>0$, there exists a $\delta>0$ such that $d_{\mathrm{S}}(A, B)<\delta$ implies $d_{\mathrm{NI}}(A, B)<\epsilon$ for all $B \in \mathcal{S}^{n}$.

This leaves us to show that the normalised indicator difference is strictly increasing. For any pair of patterns $A, B \in \mathcal{S}^{n}$, we can add a closed $n$-simplex $S$ to $B$, disjoint with $A \cup B$, resulting in a set $C \in \mathcal{S}^{n}$, with $d_{\mathrm{NI}}(A, C)>d N I(A, B)$.

\subsection{Reflection Metric}

Let $\mathcal{R}^{n}$ be the collection of subsets of $\mathbb{R}^{n}$ (not contained in any $(n-1)$ dimensional hyper-plane) that are $C^{1}$-diffeomorphic to a properly joined union of closed $(n-1)$-simplices. Formally, we write each pattern $A \in \mathcal{R}^{n}$ as $A=$ $\phi\left(\bigcup_{i=1}^{k} S_{i}\right)$, where $S_{1}, \ldots, S_{k}$ are properly joined closed $(n-1)$-simplices and $\phi \in \mathrm{C}^{1}\left(\mathbb{R}^{n}\right)$.

We use the notation $\overline{x y}$ for the open line segment connecting two distinct points $x, y \in \mathbb{R}^{n}$. We say that a point $y \in \mathbb{R}^{n}$ is visible (in $A$ ) from a point $x \in \mathbb{R}^{n}$ if $A \cap \overline{x y}=\varnothing$. For $A \in \mathcal{R}_{n}$ and $x \in \mathbb{R}^{n}$, the visibility star $V_{A}^{x}$ is defined as the set of open line segments connecting points of $A$ that are visible from $x$ :

$$
V_{A}^{x}=\bigcup\{\overline{x a} \mid a \in A \text { and } A \cap \overline{x a}=\varnothing\} .
$$

We define the reflection star $R_{A}^{x}$ by intersecting $V_{A}^{x}$ with its reflection in $x$ :

$$
R_{A}^{x}=\left\{x+v \in \mathbb{R}^{n} \mid x-v \in V_{A}^{x} \text { and } x+v \in V_{A}^{x}\right\} .
$$

Each pattern $A \in \mathcal{R}^{n}$ determines a function $\rho_{A}: \mathbb{R}^{n} \rightarrow \mathbb{R}$ given by $\rho_{A}(x)=$ $\operatorname{vol}\left(R_{A}^{x}\right)$. Note that $\rho_{A}$ is zero outside the convex hull of $A$. Figure 4 shows the visibility star $V_{A}^{x}$ and the corresponding reflection star $R_{A}^{x}$ for a pattern $A \in \mathcal{R}^{2}$, and a point $x \in \mathbb{R}^{2}$. 


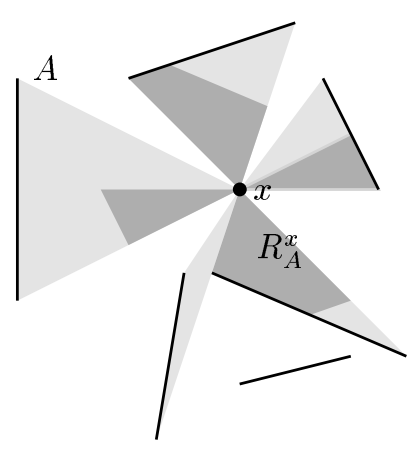

Fig. 4. Reflected visibility.

Definition 7. The reflection metric $d_{\mathrm{R}}$ for $A, B \in \mathcal{R}^{n}$ is given by

$$
d_{\mathrm{R}}(A, B)=\frac{\int_{\mathbb{R}^{n}}\left|\rho_{A}(x)-\rho_{B}(x)\right| d x}{\int_{\mathbb{R}^{n}} \max \left(\rho_{A}(x), \rho_{B}(x)\right) d x} .
$$

The reflection metric is invariant under the affine transformations $\operatorname{Af}\left(\mathbb{R}^{n}\right)$.

Observe that for any two patterns $A, B \in \mathcal{R}^{n}$ and any point $x \in \mathbb{R}^{n}$ :

$$
\operatorname{vol}\left(R_{A}^{x}-R_{B}^{x}\right) \leq 2 \operatorname{vol}\left(V_{A}^{x}-V_{B}^{x}\right)
$$

¿From this, we find that

$$
\left|\rho_{A}(x)-\rho_{B}(x)\right| \leq 2 \operatorname{vol}\left(\left(V_{A}^{x}-V_{B}^{x}\right) \cup\left(V_{B}^{x}-V_{A}^{x}\right)\right) .
$$

Thus, we can prove the first four axioms by bounding the change in the visibility star for deformation, blur, crack and noise.

The metric $d_{\mathrm{R}}$ is deformation robust. Let $A \in \mathcal{R}^{n}$, where $A=\phi\left(\bigcup_{i=1}^{k} S_{i}\right)$. Let $Q_{1}, \ldots, Q_{l}$ be the set of $(n-2)$-subsimplices of the $(n-1)$-simplices $S_{1}, \ldots, S_{k}$. Let $U_{1}$ be an open neighbourhood of $A$ with volume strictly smaller than a given number $\delta_{1}>0$. Let $L=\phi\left(\bigcup_{i=1}^{l} Q_{i}\right)$ and let $U_{2}$ be an open neighbourhood of $L$ with volume smaller than a given number $\delta_{2}>0$. Define an open neighbourhood of the identity:

$$
I=\left\{t \in \mathrm{C}^{1}\left(\mathbb{R}^{n}\right) \mid t(A) \subseteq U_{1} \text { and } t(L) \subseteq U_{2}\right\} .
$$

Given any $\epsilon>0$, we apply Eq. 14, in choosing $\delta_{1}>\delta_{2}>0$ small enough so that $d_{\mathrm{R}}(A, t(A))<\epsilon$.

The reflection metric is blur robust. Let $A \in \mathcal{R}^{n}$ and $\epsilon>0$ be given. Choose an open neighbourhood $U$ of $A$ with $\operatorname{vol}(U)<\delta$ for some given $\delta>0$. Using Eq. 14. choose $\delta>0$ small enough so that for all $B \in \mathcal{R}^{n}$ satisfying $B-U=A-U$ and $A \subseteq B$, the distance $d_{\mathrm{R}}(A, B)$ is smaller than $\epsilon$.

The reflection metric is crack robust. Let $A \in \mathcal{R}^{n}, R$ be a crack of $A$, and $\epsilon>0$. By means of Eq.14, we can choose a sufficiently small open neighbourhood 
$U$ of the crack $R$ such that $d_{\mathrm{R}}(A, B)<\epsilon$ for patterns $B \in \mathcal{R}^{n}$ satisfying $B-U=$ $A-U$.

The reflection metric is noise robust. Let $A \in \mathcal{R}^{n}$ and $x \in \mathbb{R}^{n}$ be given. Using Eq. 14, we can choose an open neighbourhood $U$ of $x$ small enough such that $d_{\mathrm{R}}(A, B)<\delta$ for all $B \in \mathcal{R}^{n}$ satisfying $B-U=A-U$.

Finally, we show that $d_{\mathrm{R}}$ is strictly increasing. Let $A, B \in \mathcal{R}^{n}$. Let $S$ be an $n$-simplex disjoint with $A$, having volume strictly larger than the convex hull of $A \cup B$. The boundary $C=\operatorname{Bd}(S)$ is a properly joined union of $(n-1)$-simplices and is therefore a member of $\mathcal{R}^{n}$. It is not difficult to see that $d_{\mathrm{R}}(A, C)>$ $d_{\mathrm{R}}(A, B)$.

\section{Conclusion}

Given a collection of patterns $\mathcal{P}$, and a group of transformations $G$, finding a metric $d$ on $\mathcal{P}$ invariant under $G$ is trivial; we can always choose the discrete metric. The real problem is finding robust metrics for a class of patterns and an invariance group. Until now, robust affine invariant pattern metrics were only known for solid sets or simple closed curves. In this paper, we introduced a robust metric, called the reflection metric, which is invariant under affine transformations, and is defined on both solid and non-solid boundaries.

We have formalised our notion of resemblance using five axioms. The axioms can be verified for any metric defined on a collection of subsets of a topological space. Axioms 14 express robustness for deformation, blur, cracks and noise, respectively. The robustness axioms guarantee that a pattern metric is wellbehaved with respect to discretisation of patterns. Axiom 5 is complementary by enforcing discriminative power on the metric.

We have used the axioms as a guiding line in analysing various metric pattern spaces. We discussed the discrete metric, the Hausdorff metric, the volume of symmetric difference and a normalised variant, the difference of normalised indicators, and the new reflection metric. Table 1 shows for collection of patterns, metrics, invariance groups, and satisfaction of axioms for each of the metric pattern spaces considered in this paper.

The reflection metric is defined on the class of finite unions of hyper-surfaces, is affine invariant, and satisfies all our axioms. This makes the reflection metric especially suitable for matching patterns obtained using image processing techniques. The reflection metric between two finite segment unions, can be computed in $O((s+k) \log (s+k)+v)$ time, where $s$ is the total number of segments, $k$ is the total number of edges in both visibility graphs, and $v$ the number of vertices in the reflection-visibility arrangement (at most $O\left(s^{2}+k^{2}\right)$ ), see $[9]$.

\section{References}

1. O. Aichholzer, H. Alt, and G. Rote. Matching shapes with a reference point. In Int. J. of Computational Geometry and Applications, volume 7, pages 349-363, August 1997. 
Table 1. Patterns, metrics, invariance, and axioms.

\begin{tabular}{cccccccc}
\hline $\mathcal{P}$ & $d$ & $G$ & Defo. & Blur & Crack & Noise & Inc. \\
\hline$\wp\left(\mathbb{R}^{n}\right)$ & $d_{\mathrm{D}}$ & $\operatorname{Hom}\left(\mathbb{R}^{n}\right)$ & no & no & no & no & no \\
$\mathcal{K}^{n}$ & $d_{\mathrm{H}}$ & $\operatorname{Iso}\left(\mathbb{R}^{n}\right)$ & yes & yes & yes & no & yes \\
$\mathcal{S}^{n}$ & $d_{\mathrm{S}}$ & $\mathrm{UJ}\left(\mathbb{R}^{n}\right)$ & yes & yes & yes & yes & yes \\
$\mathcal{S}^{n}$ & $d_{\text {NS }}$ & $\operatorname{CJ}\left(\mathbb{R}^{n}\right)$ & yes & yes & yes & yes & yes \\
$\mathcal{S}^{n}$ & $d_{\text {NI }}$ & $\operatorname{CJ}\left(\mathbb{R}^{n}\right)$ & yes & yes & yes & yes & yes \\
$\mathcal{R}^{n}$ & $d_{\mathrm{R}}$ & $\operatorname{Af}\left(\mathbb{R}^{n}\right)$ & yes & yes & yes & yes & yes \\
\hline
\end{tabular}

2. H. Alt, U. Fuchs, G. Rote, and G. Weber. Matching convex shapes with respect to the symmetric difference. In Algorithms ESA '96, Proc. 4th Annual European Symp. on Algorithms, Barcelona, Spain, September '96, pages 320-333. LNCS 1136, Springer, 1996.

3. H. Alt and M. Godeau. Computing the Fréchet distance between two polygonal curves. Int. J. of Computational Geometry $\& 5$ Applications, pages 75-91, 1995.

4. L. P. Chew, M. T. Goodrich, D. P. Huttenlocher, K. Kedem, Jon M. Kleinberg, and Dina Kravets. Geometric pattern matching under Euclidean motion. In Fifth Canadian Conference on Computational Geometry, pages 151-156, 1993.

5. S. D. Cohen and L. J. Guibas. Partial matching of planar polylines under similarity transformations. In Eight Annual ACM-SIAM Symp. on Discrete Algorithms, pages 777-786, January 1997.

6. E.T. Copson. Metric spaces. Cambridge University Press, 1968.

7. M. Hagedoorn and R. C. Veltkamp. A general method for partial point set matching. In Jean-Daniel Boissonnat, editor, Proc. 13th Annual ACM Symp. Computational Geometry, pages 406-408. ACM Press, 1997.

8. M. Hagedoorn and R. C. Veltkamp. Reliable and efficient pattern matching using an affine invariant metric. Technical Report UU-CS-1997-33, Utrecht University, 1997. revision accepted for publication in IJCV.

9. M. Hagedoorn and R. C. Veltkamp. New visibility partitions with applications in affine pattern matching. Manuscript, 1998.

10. D. P. Huttenlocher and K. Kedem. Computing the minimum Hausdorff distance for point sets under translation. In Proc. 6th Annual ACM Symp. Computational Geometry, pages 340-349, 1990.

11. D. P. Huttenlocher, K. Kedem, and M. Sharir. The upper envelope of Voronoi surfaces and its applications. Discrete and Computational Geometry, 9:267-291, 1993.

12. D. P. Huttenlocher and W. J. Rucklidge. A multi-resolution technique for comparing images using the Hausdorff distance. Technical Report 92-1321, Cornell University, 1992.

13. W. Rucklidge. Efficient Visual Recognition Using the Hausdorff Distance. Lecture Notes in Computer Science. Springer-Verlag, 1996. 